\title{
The Application of an Enhanced Recovery After Surgery to Spine Instrumentation
}

\author{
Ebru TARIKCI KILIC ${ }^{1}$, Necmi Onur TASTAN ${ }^{1}$, Caner SARIKAYA², Sait NADERI ${ }^{2}$ \\ 'Umraniye Training and Research Hospital, Department of Anesthesiology, Istanbul, Turkey \\ ${ }^{2}$ Umraniye Training and Research Hospital, Department of Neurosurgery, Istanbul, Turkey \\ Corresponding author: Ebru TARIKCI KILIC ebru.tarkc@yahoo.com
}

\section{ABSTRACT}

AIM: To evaluate the clinical and economic outcomes of the adoption of the enhanced recovery after spine surgery (ERSS) program in patients undergoing spine instrumentation.

MATERIAL and METHODS: This study described the introduction of the ERSS program, and we compared 86 consecutive patients who participated in ERSS with a retrospective cohort of 88 patients who underwent the same surgery before the implementation of this program.

Groups were compared in terms of age, sex, body mass index (BMI), American Society of Anesthesiologist (ASA) physical scores, operative time, comorbidities, intraoperative blood loss, blood transfusion rate, first oral intake, time of first mobilization, length of hospital stay, preoperative and postoperative pain scores using a numeric pain rating scale, 30-day readmission and complication rates, and total cost.

RESULTS: Groups were similar in terms of age, sex, BMI, ASA scores, and comorbidities. Intraoperative blood loss, blood transfusion rate, and length of hospital stay were lower in the ERSS group. First oral intake and first mobilization occurred earlier in the ERSS group. Postoperative pain scores were significantly lower in the ERSS group. Operative time, readmissions, or complications at 30 days did not statistically differ between the two groups. The ERSS group was found to be significantly cost effective.

CONCLUSION: ERSS is feasible, comprehensive, and cost effective for spine instrumentation with better perioperative outcomes.

KEYWORDS: Enhanced recovery, ERSS, Spine surgery, Perioperative outcomes, Cost analysis

\section{INTRODUCTION}

$\mathrm{E}$ nhanced recovery after surgery (ERAS) is a leading multimodal protocol associated with improved patient experience and perioperative outcomes with the aim of modulating and reducing the surgical stress response of patients from initial referral to discharge (3). The application of this concept of fast-track program not only improves perioperative outcomes but also reduces medical care costs (21). The goal of ERAS is based on interdisciplinary care involvements. The main components of ERAS are preoperative education, nutrition, minimally invasive surgery, opioid-sparing approach, pain management, early mobilization, early oral intake, and discharge (16).
During this process, pulmonary, cardiovascular, neurological, endocrinal, and gastrointestinal homeostasis are well maintained. In addition, optimization-oriented ERAS does not compromise patient safety or increase readmission rates.

ERAS was first adopted in colorectal surgery and has then been implemented within a number of subspecialties (7). However, despite significant data suggesting improved patient outcomes after ERAS implementation, its use in spine surgery has been limited. The current evidence from other surgical subspecialties suggests that ERAS could improve the outcome of spine surgeries for patients with lumbar degenerative diseases $(4,8)$.
Ebru TARIKCI KILIC (D) : 0000-0002-5377-1090 Necmi Onur TASTAN (D) : 0000-0002-8485-2635
Caner SARIKAYA (D): 0000-0002-2325-3903

Sait NADERI (1) : 0000-0002-6784-4270 
Morbidity associated with spine surgeries can increase, despite the improvements in anesthesia, surgical techniques, and perioperative care (12). Given the successful introduction of ERAS programs in reducing morbidity and complications and increasing the quality of life, we hypothesized that an enhanced recovery after spine surgery (ERSS) program tailored to the patients undergoing spine surgeries for instrumentation would have similar benefits.

We hypothesized that such an ERSS program would have a significant influence on clinical and economic outcomes in spine instrumentation.

\section{- MATERIAL and METHODS}

After institutional review board approval (B.10.1.TK.4.34. H.GP.0.01/159), we conducted a retrospective study at our institution in consecutive patients who underwent spine surgery for lumbar instrumentation before 1 year preceding implementation of the ERSS program and after the implementation of the ERSS in 2018.

Data were collected by manual review of the electronic medical records. All patients in the study signed written informed consentfor treatment, and all the procedures were performed in strict accordance with the principles set by the Declaration of Helsinki.

The inclusion criteria of both groups were American Society of Anesthesiologists (ASA) physical status classification of class I-III, age between 18 and 65 years, undergoing elective lumbar spine instrumentations, and with idiopathic lumbar scoliosis, degenerative spondylolisthesis, and spinal canal stenosis.

The study excluded patients with ASA IV-V physical status classification, patients younger than 18 years or older than 65 years old, patients with spinal tumors and degenerative disc diseases, and emergency surgeries consisting of acute lumbar fracture in both groups.

The groups were defined as pre-ERSS and ERSS (after the adoption of the ERSS pathway). Groups were compared in terms of age, sex, body mass index (BMI), ASA physical scores, operative time, comorbidities, intraoperative blood loss, blood transfusion rate, first oral intake, time of first mobilization, length of hospital stay, preoperative and postoperative pain scores (numeric pain rating scale [NPRS]), 30-day readmission and complication rates, and total costs. The total costs included thecost of anesthesia, surgery, intensive care unit (ICU), laboratory, and radiology for each patient.

Our primary objective was to assess the effect of ERSS application on postoperative pain scores and length of hospital stay. The secondary objective was to evaluate and analyze the total cost.

Patients in the pre-ERSS group received traditional general anesthesia with volatile agents and continuous infusion of opioids, whereas patients in the ERSS group received total intravenous anesthesia (TIVA) without opioid infusion. All the operations were performed by the same team that has 10 years of clinical experience.

\section{ERSS Group}

Patients were admitted on the same day of their operation. Based on the understanding of ERAS principles and willingness to apply, we identified a group consisting of anesthesiologists, neurosurgeons, and nurses to participate in the ERSS cases. We scheduled the same neurosurgeons, anesthesiologists, and nurses with the support of our hospital whenever possible for patient education about the ERSS program.

Preoperative education consisted of general postoperative information, enhanced recovery principles, opioid minimization pain management, and preconditioning exercises. The patients were asked to quit smoking, and antimicrobial skin cleaning was also suggested. ERAS pathway highlighted preoperative physiologic preparations, decreased stress on patients with good maintenance of physiologic function, and accelerated recovery. In accordance with the program, patients were allowed to ingest clear fluids 2 hours and solid food 4 hours before surgery. Upon arrival, gabapent in 300 $\mathrm{mg}$ with acetaminophen $1000 \mathrm{mg}$ orally were administered for preoperative pain management. Opioid medication was not considered in any of the patients. On the day of the operation, all patients received antibiotic prophylaxis 30 min before the first incision, which was in compliance with the ERAS guidelines. To prevent thromboembolism and postoperative nausea, subcutaneous low-molecular-weight heparin and intravenous $0.15 \mathrm{mg} / \mathrm{kg}$ ondansetron and $0.2 \mathrm{mg} / \mathrm{kg}$ dexamethasone were administered. TIVA was administered with bispectral index monitoring to guide anesthesia depth while maintaining hypotensive anesthesia throughout the operation. Convective warming devices were used to achieve normothermia. Tranexamic acid (TXA) was used both intravenously and topicallyto achievea conservative blood management strategy. Intravenous $1.5 \mathrm{~g}$ TXA was administered in 100 $\mathrm{mL}$ normal saline during surgery, and another $1 \mathrm{~g}$ TXA was administered topically in $100 \mathrm{~mL}$ normal saline during suturing at the end of the operation. Fluid management was restricted as well as blood transfusions based on surgical and patient risk factors. The utilization of blood products was minimized, and transfusion was performed only if hemoglobin is $<8 \mathrm{~g} /$ $\mathrm{dL}$, depending on comorbidities. Marcaine hydrochloride $0.5 \%$ (30 cc) was infiltrated into the subcutaneous tissues after wound closure. Extubation was performed at the end of the surgery in the operating room. ICU admissions were minimized. Minimally invasive surgery (MIS) techniques were preferred when feasible. Nasogastric tubes, urinary catheters, and drains were not utilized.

In the postoperative period, an opioid-sparing approach was adopted. A numeric rating scale was used to classify pain intensity. Acetaminophen and nonsteroidal anti-inflammatory drugs were administered for NPRS $>4$, where as tramadol was used as a rescue medication for NPRS $>8$. Early ambulation is a key component of ERAS and was planned in the ERSS group. Mobilization was encouraged on the day of the surgery with at least some movements three times daily and food and drink intake as soon as possible. Patients were discharged when they were able to start daily activities and otherwise ready for discharge. 


\section{Pre-ERSS Group}

Patients were admitted on the same day of the operation. The perioperative care and preoperative counseling was according to the surgeon's preference. Antibiotic prophylaxis was administered 30 minutes before taking the patient to the operating room. Thromboembolism and antiemetic prophylaxis were not used. Fasting time for clear fluid was 4 hours and solid food 8 hours before surgery.

Patients received a conventional balanced general anesthesia using a combination of volatile agents, intravenous anesthetics, and opioid in fusion. Fluid management and blood product utilization depended on the individual provider's preference. Temperature management was not performed. Urinary catheters and drains were utilized. At the end of the surgery, patients were admitted and extubated in the ICU unit. In the postoperative period, when NPRS $>4,100 \mathrm{mg}$ of pethidine was used intravenously. Morphine was injected intravenously if NPRS > 8. On the second postoperative day, patients were discharged to a regular ward. Clear liquids were started and, diet was advanced as tolerated. Patients were discharged when they were able to start daily activities and otherwise ready for discharge.

\section{Surgical Procedure}

Surgery was performed in prone position. To reduce intraabdominal pressure and intraoperative bleeding, two surgical pillows were placed between the thorax and pelvis. After fluoroscopic imaging of the surgical site, the lumbar area was prepared and draped. A midline skin incision from cranial to the caudal spine was made, and after opening, the fascia in the midline paravertebral muscles were dissected bilaterally. All the screws were placed in the pedicle under fluoroscopic control. After placement of all screws, lateral and anteroposterior X-rays were taken. Based on the type of pathology, a hemi- or total laminectomy was performed under a microscope vision. Discectomy was performed. For fusion purpose, a transforminal lumbar interbody fusion of posterior lumbar interbody fusion cages, filled with autologous bone grafts, were placed. After placement of the cages, all the screws were fixated using compression mode. In the ERSS group, the fascia, subcutaneous tissue, and the skin were closed after hemostasis; $1 \mathrm{~g}$ TXA in $100 \mathrm{~mL}$ of normal saline was used topically while suturing. Marcaine hydrochloride $0.5 \%$ (30 cc) was infiltrated into the subcutaneous tissues after wound closure. In the ERSS group, drains or urinary catheters were not utilized.

\section{Statistical Method}

SPSS 17.0 for windows was used to analyze collected data. Analyses were performed at 0.05 alpha and $95 \%$ confidence interval. Nominal and ordinal data were described by frequencies, whereas scale parameters were described as mean and standard deviations (SD). Non-normal distributed data were also described as mean and SD to compare parameter effects. Kolmogorov-Smirnov with Lilliefors correction and Shapiro-Wilk tests were used for the normality of parameters. Since all the parameters were found to be nonparametric, Mann-Whitney $U$ and chi-square tests were used for differential analysis.

\section{RESULTS}

Of the 174 patients included, 88 patients were in the pre-ERSS group, and 86 patients were in the ERSS group. Demographic data and descriptive characteristics of the patients are shown in Table I. There were no significant differences between the two groups concerning age, sex, BMI, and ASA scores $(p>0.05)$. Chronic cardiovascular disease, chronic pulmonary disease, and diabetes mellitus did not differ between the two groups $(p>0.05)$. The ERSS group had a tendency of shorter operation time $(4.56 \pm 0.62 \mathrm{~h})$ than the pre-ERSS group (4.70 $\pm 0.96 \mathrm{~h}$ ) but did not differ statistically $(\mathrm{p}=0.244)$.

Table I: Demographic Characteristics in the Pre - ERSS and ERSS Groups

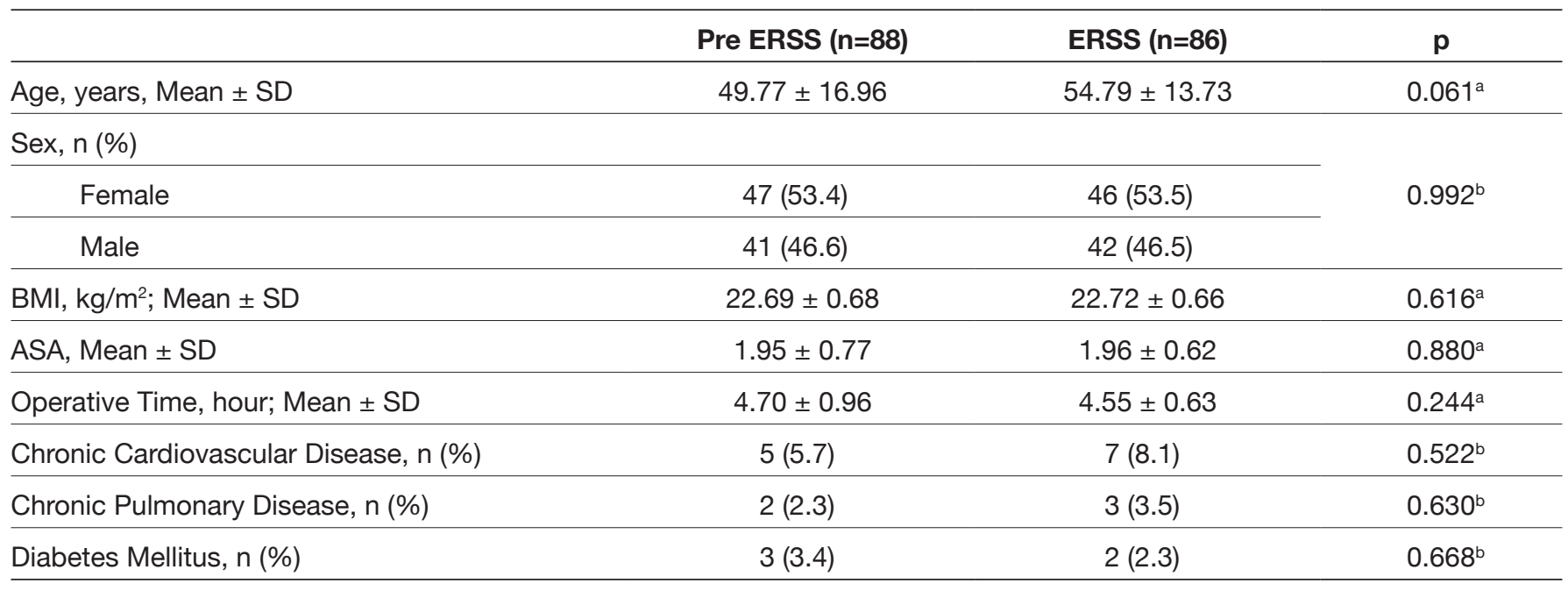

a: Mann -Whitney-U test, b: Chi-Square Test. BMI: Body mass index, ASA: American Society of Anesthesiologists. Data are presented as mean $\pm S D$ unless otherwise indicated. 
Some clinical and hospitalization results of both patient groups are given in Table II.

Intraoperative blood loss was significantly lower in the ERSS group (204.42 $\pm 124.40 \mathrm{~mL})$ compared with the pre-ERSS group (414.26 $\pm 237.64 \mathrm{~mL} ; \mathrm{p}<0.05)$. Similarly, the blood transfusion rate was significantly lower in the ERSS group $(1.08 \pm 0.29$ unit) than in the pre-ERSS group (2.00 \pm 0.92 unit; $\mathrm{p}<0.05)$. First oral intake and first mobilization were earlier in the ERSS group ( $4.34 \pm 0.85$ vs. $8.82 \pm 3.41 \mathrm{~h}$, respectively). The length of hospital stay was also lower in the ERSS group $(\mathrm{p}<0.05)$.

Preop and Postop NPRS levels of patients are given in Table III.

Preoperative NPRS scores did not differ significantly between both groups $(p=0.088)$. However, postoperative NPRS scores were significantly higher in the pre-ERSS group $(p<0.000)$.

Readmission and complication rates within 30 days of the patients are shown in Table IV.

There was no 30-day mortality in both groups. Readmission and complication rates within 30 days were lower in the ERSS group but did not differ statistically $(p>0.540)$. The overall complications were cardiovascular, respiratory, neurologic, infectious, or renal.
Hospitalization costs are shown in detail in Table V. The cost of anesthesia, surgery, ICU stay, laboratory, and radiology were significantly lower in the ERSS group compared with the preERSS group $(\mathrm{p}<0.05)$.

Indications for pedicle screw instrumentation are shown in Table VI. The two groups did not differ regarding the indications for pedicle screw instrumentation statistically ( $p>0.05)$.

\section{DISCUSSION}

The adoption of ERAS in spinal surgeries is limited but certainly offers better patient outcomes with a fast-track recovery. We observed that the ERAS program improved the perioperative outcomes and postoperative pain scores and shortened the length of hospital stay. ERAS application was significantly cost effective and trended toward better meaningful recovery.

Our ERAS program included educating preoperative patient, encouraging patient participation that we believe contributes to early recovery, and defining clearly the expectations for relieving the emotional stress and anxiety. Patient education was reinforced regarding the use of analgesics, highlighting that acetaminophen and nonsteroidal anti-inflammatory should be the first choice before starting an opioid analgesic.

Table II: Intraoperative and Postoperative outcomes of Both Groups

\begin{tabular}{lccc}
\hline Parameters, Mean \pm SD & Pre-ERSS $(\mathbf{n = 8 8})$ & ERSS $(\mathbf{n = 8 6 )}$ & $\mathbf{p}$ \\
\hline Blood Loss, ml & $414.26 \pm 237.64$ & $204.42 \pm 124.40$ & $1.08 \pm 0.29$ \\
\hline Transfusion, unit & $2.00 \pm 0.92$ & $4.34 \pm 0.85$ & $<0.05^{\mathrm{a}}$ \\
\hline First oral intake, hour & $8.82 \pm 3.41$ & $13.80 \pm 1.41$ & $<0.05^{\mathrm{a}}$ \\
\hline First Mobilization, hour. & $25.40 \pm 3.13$ & $31.24 \pm 4.87$ & $<0.05^{\mathrm{a}}$ \\
\hline Length of hospital stay, hour & $49.52 \pm 5.96$ & $<.05^{\mathrm{a}}$ \\
\hline
\end{tabular}

a: Mann - Whitney-U Test.

Table III: Preop and Postop NPRS Scores

\begin{tabular}{lccc}
\hline Parameters, Mean \pm SD & Pre-ERSS $(\mathbf{n = 8 8})$ & ERSS $(\mathbf{n = 8 6 )}$ & $\mathbf{p}$ \\
\hline Preop NRS & $6.65 \pm 0.69$ & $6.50 \pm 0.81$ & $0.088^{\mathrm{a}}$ \\
\hline Postop NRS (12 Hours) & $4.65 \pm 1.41$ & $1.84 \pm 0.96$ & $\mathbf{0 . 0 0 0 ^ { \mathrm { a } }}$ \\
\hline Postop NRS (24 Hours) & $4.48 \pm 1.31$ & $1.74 \pm 0.81$ & $\mathbf{0 . 0 0 0 ^ { \mathrm { a } }}$ \\
\hline
\end{tabular}

a: Mann - Whitney-U Test.

Table IV: Readmission and Complication Rates within 30 Days After Surgery

\begin{tabular}{lccc}
\hline Parameters, Mean \pm SD & Pre-ERSS $(\mathbf{n = 8 8 )}$ & ERSS (n=86) & $\mathbf{p}$ \\
\hline 30 Day Readmission Rate & $1.54 \pm 0.76$ & $1.48 \pm 0.85$ & $0.255^{\mathrm{a}}$ \\
\hline 30 Day Complication Rate & $13(14.8)$ & $10(11.6)$ & $0.540^{\mathrm{b}}$ \\
\hline
\end{tabular}

a: Mann - Whitney-U Test, b: Chi-Square Test. 
Table V: Hospitalization costs in the pre ERSS and ERSS groups

\begin{tabular}{lccc}
\hline Variable, Mean \pm SD & Pre-ERSS $(\mathbf{n = 8 8})$ & ERSS (n=86) & p \\
\hline Cost of Anesthesia, TL & $533.86 \pm 19.56$ & $232.32 \pm 19.44$ & $<.05^{\mathrm{a}}$ \\
\hline Cost of Surgery, TL & $3517.23 \pm 848.33$ & $3494.19 \pm 931.46$ & $0.830^{\mathrm{a}}$ \\
\hline Cost of Hospital Stay with ICU, TL & $4994.09 \pm 847.31$ & $3726.51 \pm 934.70$ & $<\mathbf{0 . 0 5 ^ { \mathrm { a } }}$ \\
\hline Laboratory Cost, TL & $383.64 \pm 18.39$ & $279.30 \pm 16.43$ & $<\mathbf{0 . 0 5 ^ { \mathrm { a } }}$ \\
\hline Radiology Cost, TL & $407.16 \pm 49.31$ & $271.98 \pm 13.36$ & $<\mathbf{0 . 0 5 ^ { \mathrm { a } }}$ \\
\hline
\end{tabular}

a: Mann - Whitney-U Test, ICU: Intensive Care Unit, TL: Turkish Lira.

Table VI: Indication for Pedicle Screw Instrumentation

\begin{tabular}{lcc}
\hline & Pre-ERSS (n=88) & ERSS (n=86) \\
\hline Idiopathic lumbar scoliosis & $4(4.5)$ & $3(3.4)$ \\
\hline Degenerative spondylolisthesis & $24(27.3)$ & $20(22.7)$ \\
\hline Spinal canal stenosis & $60(68.2)$ & $63(73.9)$ \\
\hline Total & $88(100.0)$ & $86(100.0)$ \\
\hline
\end{tabular}

a: Chi-Square Test.

In a study of 175 patients undergoing spine surgery, Lee et al. showed that $87 \%$ of the patients expressed preoperative anxiety attributed to the fear of anesthesia and surgery. In their study, they reported that trust in the medical staff and the team's explanation of the details about the operation were the most helpful factors in overcoming preoperative catastrophic thinkings with unnecessary anxiety $(1,5,14)$.

Papanastassiou et al. stated that a group of spinal surgery patients, who participated in a presurgery class, had better satisfaction and pain control (96\% vs. $83 \%)$ compared with patients who did not attend ERAS (20).

Our first aim was to reduce opioid consumption. Anesthetic management included administering TIVA of propofol intraoperatively and acetaminophen intravenously to maintain hypotensive anesthesia and infiltrating wound infiltrative bupivacaine hydrochloride into the wound (13). Intravenous dexamethasone was used for postoperative nausea and vomiting. TXA was an effective strategy for reducing blood loss and transfusion rate $(22,26)$. We maintained intraoperative normovolemia by restricting fluid administration and followed the ERAS guidelines for blood transfusion by avoiding transfusion below the hemoglobin levels of $8 \mathrm{~g} / \mathrm{dL}$. Administering prophylaxis before the operation, giving appropriate antibiotic 30 min before the first incision, and achieving target normothermia in the operating room were the main components in our protocol.

The primary outcome in this study was postoperative pain management and length of hospital stay due to surrogate for recovery and to test the hypothesis that ERAS is a useful protocol to facilitate spine surgery.
In this study, local anesthetics, steroids, acetaminophen, and gabapentinoids were scheduled for postoperative pain control. Opioid-sparing pain management reduced the related side effects such as nausea vomiting, ileus, sedation, and respiratory failure. Although postoperative hospital stay depends on patient-related factors such as age, comorbidities, pain, and immobilization and can be influenced by the discharge policies of the hospital, we demonstrated a significant reduction in the length of hospital stay in the ERSS group.

In the ERSS group, allied strictly with the ERAS guidelines, we observed a significantly shorter operative time because of the minimally invasive surgical techniques and TIVA usage leading to hypotensive anesthesia, with better pain relief, shorter hospital stay, and lower readmission scores and complication rates even though the two study groups have similar demographic aspects, comorbidities, and ASA scores.

Many systematic reviews and studies suggest minimally invasive approaches for spine surgeries due to the associated benefits like less blood loss, less pain, shorter operative time, shorter recovery period, and reduced cost $(10,15,19)$. The advances in technology and several improvements in surgical specialties and the minimally invasive tissue-protecting techniques increased the peri-postoperative outcomes. Therefore, a meaningful combination of the ERAS concept with minimal invasive surgery is strongly suggested.

The present study demonstrated an economic benefit related to the ERAS program. The decreased postoperative morbidity, length of hospital stay, and patients directly transferring to the ward avoiding ICU stay were the main components of the cost effectiveness. Similarly, costs related to the operation, 
anesthesia, laboratory, and radiology costs were significantly lower in the ERSS group compared with the pre-ERSS group.

In our ERSS group, preoperative education introduced the aim and procedure of the ERSS protocol, pain coping strategies, and operation details. Pain control was better in the ERSS group than in the traditional care group in terms of mean NPRS score and mean maximum NPRS score after surgery.

Mathiesen et al., in a retrospective study, reported better pain scores with less opioid consumption leading to faster mobilization after implementing ERAS protocols in multilevel spine surgeries (18).

In another study, Wang et al. compared MISS versus open surgery for spinal fusion using ERAS (25). The results were associated with lower blood loss and reoperation rates.

Wainwright et al. supported the adoption of ERAS pathways and reported that ERAS fastened the recovery period with lower rates of morbidity and improved the long-term results (23).

Grasu et al. described ERAS in patients undergoing spine surgery for tumor metastasis and compared 41 patients in ERSS with the retrospective data of 56 patients before the implementation of the ERAS program. They showed that ERAS was effective inpostoperative pain, length of hospital stay, 30-day readmission, and 30-day complication rate (9).

Adogwa et al. conducted another study for the adoption of the ERAS program for idiopathic scoliosis surgery that resulted in rapid recovery, faster mobilization, and earlier discharge (2).

Fleege et al. introduced a fast-track protocol for patients undergoing stabilization for degenerative spine pathologies. They reported a reduced length of hospital stay with better patient satisfaction (6).

Jun et al. retrospectively reviewed the medical records of patients who underwent cervical laminoplasty for degenerative multilevel spine compression and spinal canal stenosis. They reported better scores in the first time of mobilization, first oral intake, and time of discharge from the hospital (11).

Mobilizing and getting out of bed "early" reportedly reduced the duration of hospital stay and improved the postoperative functional status in spine surgeries. Delayed ambulation can be associated with higher complication rates, such as deep venous thrombosis, pulmonary embolism, atelectasis, stroke, and others (24).

Reducing fasting time before surgery leads to a decrease in anxiety and starvation-induced insulin resistance. In our study, patients in the ERSS group were able to drink fluids within 2 hours after surgery and can consume a liquid diet after 4 hours. This practice reduces postoperative cardiorespiratory complications and shortens the length of hospitalization $(15,17)$.

In our study, the complication rate was lower in the ERSS group but did not differ statistically between the two groups, probably because of the small sample size. Larger and longterm follow-up is needed.
At present, the main barrier that hinders the successful adoption of ERAS is the reluctance to change; therefore, healthcare providers must believe the importance of innovation in perioperative care and treatment.

\section{CONCLUSION}

ERAS is a quality implementation program that depends on culture, systematic data, information systems, and approaches that are well designed. The use of ERAS guidelines allows the surgical teams to understand and facilitate perioperative care with the goal to improve the patient's outcomes.

ERAS protocols are beneficial, feasible, and based on our experience, can be easily adopted in spine surgery. Future studies with larger groups of patients are needed to strengthen our reported outcomes. ERAS pathways should be adopted by spine care teams.

\section{REFERENCES}

1. Adogwa O, Elsamadicy AA, Cheng J, Bagley C: Pretreatment of anxiety before cervical spine surgery improves clinical outcomes: A prospective, single-institution experience. World Neurosurg 88:625-630, 2016

2. Adogwa O, Elsamadicy AA, Fialkoff J, Cheng J, Karikari IO, Bagley C: Early ambulation decreases length of hospital stay, peri-operative complications and improves functional outcomes in elderly patients undergoing surgery for correction of adult degenerative scoliosis. Spine (Phila Pa 1976) 42: 1420-1425, 2017

3. Boltz MM, Hollenbeak CS, Ortenzi G, Dillon PW: Synergistic implications of multiple postoperative outcomes. Am J Med Qual 27:383-390, 2018

4. Deyo RA, Mirza SK, Martin BI, Kreuter W, Goodmann DC, Jarvik JG: Trends, major medical complications, and charges associated with surgery for lumbal spinal stenosis in older adults. JAMA 303:1259-1265, 2010

5. Elsamadicy AA, Adogwa O, Cheng J, Bagley C: Pretreatment of depression before cervical spine surgery improves patients' perception of postoperative health status: A retrospective, single institutional experience. World Neurosurg 87:214-219, 2016

6. Fleege C, Almajali A, Rauschmann M, Rickert M: Improve of surgical outcomes in spinal fusion surgery: Evidence based peri-and intra-operative aspects to reduce complications and earlier recovery. Der Orthopäde 43:1070-1078, 2014

7. Gotlib Conn L, McKenzie M, Pearsall EA, McLeod RS: Successful implementation of an enhanced recovery after surgery programme for elective colorectal surgery: A process evaluation of champions' experiences. Implement Sci 10:99, 2015

8. Grant MC, Hanna A, Benson A, Hobson D, Wu CL, Yuan CT, Rosen M, Wick EC: Dedicated operating room teams and clinical outcomes in an enhanced recovery after surgery pathway for colorectal surgery. J Am Coll Surg 226:267-276, 2018 
9. Grasu MR, Cata PJ, Dang QA, Tatsui EC, Rhines DL, Hagan BK, Bhavsar S, Raty SR, Arunkumar R, Potylchansky Y, Lipski I, Arnold BA, McHugh TM, Bird JE, Rodriguez-Restrepo A, Hernandez M, Popat KU: Implementation of an enhanced recovery after spine surgery program at a latge cancer center: A preliminary analysis. J Neurosurg Spine 29:588-598, 2018

10. Joliat GR, Labgaa I, Petermann D, Hübner M, Griesser $A C$, Demartines N, Schäfer M: Cost-benefit analysis of an enhanced recovery protocol for pancreaticoduodenectomy. Br J Surg 102:1676-1683, 2015

11. Jun L, Hao L, Zheng Kuan Xv, Jian W, Qun Fei Y, Gang C, Fang-Cai L, Ying R, Qi-Xin C: Enhanced recovery care versus traditional care following laminoplasty. A retrospective casecohort study. Medicine (Baltimore) 97(48): e13195, 2018

12. Kanaan SF, Waitman LR, Yeh HW, Arnold PM, Burton DC, Sharma NK: Structural equation model analysis of the length -of -hospital stay after lumbar spine surgery. Spine J 15:612621,2015

13. Kim J, Burke SM, Kryzanski JT, Roberts RJ, Roguski M, Qu E, Hwang SW, Liu PP, Desilier A, Riesenburger RI: The role of liposomal bupivacaine in reduction of post-operative pain after transforaminal lumbar interbody fusion (TLIF): A clinical study. World Neurosurg 91:460-467, 2016

14. Lee JS, Park YM, Ha KY, Cho SW, Bak GH, Kim KW: Preoperative anxiety about spinal surgery under general anesthesia. Eur Spine J 25:698-707, 2016

15. Ljungvist $\mathrm{O}$, Hubner $\mathrm{M}$ : Enhanced recovery after surgeryERAS principles, practise, and feasibility in the elderly. Aging Clin Exp Res 30:249-252, 2018

16. Ljungqvist $O$, Scott $M$, Fearon KC: Enhanced recovery after surgery: A review JAMA Surg 152:292-298, 2017

17. Martin D, Roulin D, Grass F, Addor V, Ljungquvist O, Demartines $\mathrm{N}$ : A multicentre qualitative assessing implementation of an enhanced recovery after surgery program. Clin Nutr 37:21722177,2018
18. Mathiesen O, Dahl B, Thomsen BA, Kitter B, Sonne N, Dahl $\mathrm{JB}$, Kehlet $\mathrm{H}$ : A comprehensive multimodal pain treatment reduces opioid consumption after multilevel spine surgery. Eur Spine J 22:2089-2096, 2013

19. Nicholson A, Lowe MC, Parker J, Lewis SR, Alderson P, Smith AF: Systematic review and meta-analysis of enhanced recovery programmes in surgical patients. Br J Surg 101:172188,2014

20. Papanastassiou I, Anderson R, Barber N, Conover C, Castellvi $A E$ : Effects of preoperative education on spinal surgery patients. SAS J 5:120-124, 2011

21. Roulin D, Donadini A, Gander S, Griesser AC, Blanc C, Hübner $M$ : Cost effectiveness of the implementation of an enhanced recovery protocol for colorectal surgery. Br J Surg 100:11081014, 2013

22. Tarikci Kilic E, Demirbilek T, Naderi S: Does an enhanced recovery after surgery protocol change costs and outcomes of single-level lumbar microdiscectomy? Neurosurgical Focus 46(4)1-6, 2019

23. Wainwright TW, Immins T, Middleton RG: Enhanced recovery after surgery (ERAS) and its applicability for major spine surgery. Best Pract Res Clin Anaesthesiol 30:91-102, 2016

24. Wang D, Teddy PJ, Henderson NJ, Shine BS, Gardner BP: Mobilization of patients after spinal surgery for acute spinal cord injury. Spine (Phila Pa 1976) 26:2278-2282, 2001

25. Wang MY, Chang PY, Grossman J: Development of an enhanced recovery after surgery (ERAS) approach for lumbar spinal fusion. J Neurosurg Spine 26:411-418, 2017

26. Zhang F, Wang K, Li FN, Huang X, Li Q, Chen Z, Tang YB, Shen HX, Song QX: Effectiveness of tranexamic acid in reducing blood loss in spinal surgery: A meta analysis. BMC Musculoskeletal Disord 15:448-456, 2014 